\title{
Dilatonic Entropic Force
}

\author{
I.Sakalli* \\ Department of Physics, Eastern Mediterranean University, \\ G. Magusa, north Cyprus, Mersin-10, Turkey and \\ *izzet.sakalli@emu.edu.tr
}

\begin{abstract}
We show in detail that the entropic force of the static spherically symmetric spacetimes with unusual asymptotics can be calculated through the Verlinde's arguments. We introduce three different holographic screen candidates, which are first employed thoroughly by Myung and Kim [Phys. Rev. D 81, 105012 (2010)] for Schwarzschild black hole solutions, in order to identify the entropic force arising between a charged dilaton black hole and a test particle. The significance of the dilaton parameter on the entropic force is highlighted, and shown graphically.
\end{abstract}

\section{INTRODUCTION}

Since Einstein's epochal studies [1] on general relativity (GR), it has been argued that gravity is different at a deep level than other fundamental forces. According to the GR, one should think of gravity as a deformation of spacetime due to the presence of energy or stress. Although, in the appropriate limit, GR reduces to Newtonian gravity, till the present day there is no confirmed evidence for the gravitational fields, and their source - graviton. Because of this, whenever the agenda is about gravity, new theories continue to attract much attention in physics. Recently, Verlinde [2] has invented a conceptual theory that gravity is emergent, rather than the fundamental force. Essentially, Verlinde showed that one can start from very general considerations, and gravity emerges as an entropic force. In addition to this, Verlinde's arguments reveal a fact that the key to understanding gravity is information (or entropy).

It is obvious that Verlinde was highly inspired by early works on information storage in black holes by Bekenstein [3], Hawking [4] and 't Hooft [5]. In the framework of quantum gravity, such an information storage is explained best by the holographic principle [5, 6], which was originally proposed by 't Hooft [5]. In fact, the holographic principle states that the entropy of a black hole $(\mathrm{BH})$ is proportional to the surface area of its event horizon and not its volume; that volume itself is illusory and the universe is really a hologram which is isomorphic to the information inscribed on the surface of its boundary. Namely, the amount of information needed to describe a BH can be entirely coded on its event horizon. Verlinde, considering both holographic principle and the second law of thermodynamics 3], showed that an entropic force is an apparent force that results from changes in the information associated with the positions of particles such that it drags particles towards and across the event horizon. Videlicet, entropic forces are at work to pull particles across the holographic screen. Macroscopically, this effect is nothing but the gravity. Meanwhile, it should be noted that, prior to the Verlinde's work, Padmanabhan [7] also obtained the Newton's law of gravity by using the equipartition law of energy; $E=2 T S$. However, in addition to the Newton's second law and Newtonian gravity, the astounding contribution coming from Verlinde is the derivation of the Einstein's equations. Soon after Verlinde's new proposal about the gravity, many relevant works appeared. Some related (and very recently published) works can be seen in [8], and references therein.

In this paper, we think that it is interesting to consider the derivation of Verlinde's entropic force in a spacetime with unusual asymptotics. To this end, we would like to contribute to the literature, and try to learn more about the concept of entropic force. Because, we believe that only when more and more results on entropic force are available, Verlinde's arguments will be more reliable. The paradigm of spacetimes with unusual asymptotics is best described by the metric of charged dilaton black holes $(\mathrm{CDBHs})$ 9]. In this paper we shall be concerned with CDBHs, which are stationary and static. CDBHs have both asymptotically flat (AF) and non-asymptotically flat (NAF) features depending on their dilaton parameter - " $a$ ". The beauty of CDBHs is that they have Schwarzschild limit when the dilaton field vanishes. Here, we introduce three different candidates in order to define the holographic screen (HS), locating at equipotential surfaces, of a CDBH: accelerating surface (AS), static HS and stretched horizon (SH), which might also be described as a Rindler spacetime. After giving the brief summary of formulation of the entropic force, we calculate the each temperature of these holographic screens, and plug the temperatures separately into the derived entropic force formula. In doing this, we also verify that the obtained entropic forces reduce to the results found in a very recent study [10], by Myung and Kim, in which the Schwarzschild spacetime is considered, and thus to the Newton's force law. Of course, the ultimate aim here is to highlight the effect of the dilaton parameter on the entropic force by making use of numerical computation and plotting.

The outline of the present paper is as follows. A brief summary of the idea underlying Verlinde's entropic force is given in the next section. Sec. III and Sec. IV are devoted to the derivation of the entropic force by using AS and static HS methods, respectively. Sec. V, we calculate the entropic force on the SH. The paper ends with a conclusion, which appears in Sec. VI. 
Throughout the paper, the natural units of $c=G=k_{B}=\hbar=1$ are used.

\section{A BRIEF SUMMARY OF ENTROPIC FORCE}

Here we briefly mention some key points of the entropic force by following [2]. As a demonstration to the usage of Verlinde's entropic force, we retrieve the Newton's law of gravitation by using the holographic screen in the nonrelativistic case.

Verlinde postulated that the change of entropy on a holographic screen in which a test particle of mass $m$ is located very close to it is linear with distance $\Delta x$, namely

$$
\Delta S=2 \pi m \Delta x
$$

According to the first law of thermodynamics, the entropic force exerting on the test particle due to the change in entropy is

$$
F \Delta x=T \Delta S
$$

where $T$ is the temperature. After manipulating Eqs. (1) and (2), it is easy to see that

$$
F=2 \pi m T
$$

From here on, above identity will be our master equation to obtain the entropic force. Simply put, once the temperature on the holographic screen is defined, we just insert it into Eq. (3) and get the entropic force.

Remarkably, let us recall the equipartition rule of energy:

$$
E=\frac{1}{2} \tilde{N} T
$$

where $\tilde{N}$ is the partition number. As proposed by Verlinde, if we combine the holographic principle and the equipartition rule of energy, $\tilde{N}$ is interpreted as the number of bits on the holographic screen. In fact, this number is proportional to the area of the holographic screen as

$$
\tilde{N}=c A,
$$

where $c$ is an constant, which is equal to one for AF and spherically symmetric spacetimes. As it can be seen in the latter section, $c$ can take values different than one depending on the non-asymptotic flatness of the spacetime.

In order to reach the Newtonian force law, it is plausible to assume the holographic screen as a spherical screen with radius $R$ which belongs to an AF spacetime, whose its total mass $M$ is located at its centre. Thus, the equipartition of energy rule takes the following form

$$
\begin{aligned}
E & =M=\frac{1}{2} A T, \\
& =2 S T,
\end{aligned}
$$

where $S=\frac{A}{4}$ is the entropy of the holographic screen with the area $A=4 \pi R^{2}$. It is needless to say that $E$ is identified with the mass $M$ inside the screen. By using Eq. (6), one can easily read the temperature on the holographic screen as

$$
T=\frac{M}{2 \pi R^{2}},
$$

As we emphasized before, once the temperature of the holographic screen is determined, the entropic force is easily read from Eq. (3). Thus, after plugging Eq. (7) into Eq. (3), we obtain the entropic force as

$$
F_{N}=\frac{M m}{R^{2}}
$$

which is the well-known Newton's law of gravitation. 


\section{FEATURES OF CDBH}

In the low-energy limit of string field theory, the four-dimensional action (in Einstein frame) describing the dilaton field $\phi$ with its parameter $a$ (without loss of generality, we consider $a>0$ ) coupled to a $U(1)$ gauge field [11] is

$$
S=\int d^{4} x \sqrt{-g}\left(\Re-2(\nabla \phi)^{2}-e^{-2 a \phi} F^{2}\right)
$$

where $\Re$ is the curvature scalar and $F^{2}=F_{\mu v} F^{\mu v}$ in which $F_{\mu v}$ is the Maxwell field associated with a $U(1)$ subgroup of $E_{8} \times E_{8}$ or $\operatorname{Spin}(32) / Z_{2}$. In the presence of a electric charge with the non-constant the dilaton field, the static spherically symmetric solutions are designated with CDBHs [9] whose metric is given by

$$
d s^{2}=-f d t^{2}+\frac{d r^{2}}{f}+R^{2}\left(d \theta^{2}+\sin ^{2} \theta d \varphi^{2}\right)
$$

where the metric functions $f$ and $R$ are

$$
f=\frac{1}{\gamma^{2}} r^{\frac{2}{1+a^{2}}}\left(1-\frac{r_{+}}{r}\right)
$$

and

$$
R=\gamma r^{N}
$$

Here $r_{+}$denotes the event horizon of the CDBHs, $\gamma$ is an arbitrary real constant, and another real constant $N$ is governed by

$$
N=\frac{a^{2}}{1+a^{2}}
$$

The dilaton is found in the following form

$$
\phi=\phi_{0}+\phi_{1} \ln r
$$

where

$$
\phi_{0}=-\frac{1}{2 a} \ln \left[\frac{Q^{2}\left(1+a^{2}\right)}{\gamma^{2}}\right] \text { and } \phi_{1}=\frac{N}{a},
$$

where $Q$ refers to the electric charge. In this case, the solution for the electromagnetic field is found as

$$
F_{t r}=\frac{Q e^{2 a \phi}}{R^{2}}
$$

CDBHs are not vacuum solutions since the action (9) contains a static dilaton fluid whose energy-momentum tensor never vanishes. Essentially, taking account of such a particular fluid model makes the CDBHs so special that they are neither AF nor NAF.

By following the quasilocal mass definition for the NAF BHs, which was defined by Brown and York [12], one can see that the horizon $r_{+}$is related to the mass $M$ through

$$
r_{+}=\frac{2 M}{N}
$$

For $r_{+}>0$, the horizon at $r=r_{+}$hides the singularity located at $r=0$. In the extreme case $r_{+}=0$, metric (10) still exhibits the features of the BHs. Because the singularity at $r=0$ is null and marginally trapped such that it prevents the outgoing signals to reach the external observers. Besides all these, a CDBH has no inner horizon and no extremal limit on the charge $Q$. This is one of the characteristics of the CDBHs. Metric functions (11) and (12) correspond to the linear dilaton BHs [13] in the case of $a=1$. On the other hand, while $a \rightarrow \infty$ with $\gamma=1$, metric (10) reduces to the Schwarzschild BH. Finally, it is worth to note that the CDBHs have no zero charge limit. 


\section{ACCELERATING SURFACE OF CDBH AND THE ENTROPIC FORCE}

The aim of this section is to define the entropic force on the AS of the CDBH. The definition of the AS or so-called bulk is first introduced by Mäkelä [14], and it has been recently used in [10] in order to derive the entropic force appeared in the Schwarzschild BH. Here, we also consider the AS as if a HS, and we shall derive the entropic force for the CDBHs.

If one defines a future pointing unit vector $u^{\alpha}$, which is the congruence for the timelike world lines of the points on a spacelike hypersurface $S^{2}$, it should satisfy the following orthogonality condition

$$
u^{\alpha} n_{\alpha}=0
$$

where $n_{\alpha}=\left[0, \frac{1}{\sqrt{f}}, 0,0\right]$ is a normal vector on $S^{2}$. Generic choice of the future pointing unit vector is $u^{\alpha}=$ $\left[\frac{1}{\sqrt{f}}, 0,0,0\right]$. Essentially, the reason of using the future pointing unit vector $u^{\alpha}$ is to find the change of the heat, which is related with the acceleration. Thus, it is plausible to introduce the local acceleration in terms of $u^{\alpha}$ as follows:

$$
\tilde{a}^{\mu}=u^{\alpha} u_{; \alpha}^{\mu}
$$

where "; denotes the covariant derivative. This implies that the only nonzero component of the proper acceleration vector is

$$
\tilde{a}^{r}=\frac{f^{\prime}}{2}=\frac{1}{\gamma^{2}\left(1+a^{2}\right)} r^{\frac{1-a^{2}}{1+a^{2}}}\left[1+\frac{r_{+}\left(a^{2}-1\right)}{2 r}\right],
$$

in which a prime symbol denotes derivative with respect to $r$. The proper acceleration is defined by

$$
\tilde{a}=\tilde{a}^{\mu} n_{\mu}
$$

which yields

$$
\tilde{a}=\frac{r^{-N}}{\gamma\left(1+a^{2}\right)} \frac{\left[1+\frac{r_{+}\left(a^{2}-1\right)}{2 r}\right]}{\sqrt{1-\frac{r_{+}}{r}}},
$$

By the virtue of the above result, one can read the Unruh temperature [15] as

$$
T_{U}=\frac{\tilde{a}}{2 \pi}=\frac{r^{-N}}{2 \pi \gamma\left(1+a^{2}\right)} \frac{\left[1+\frac{r_{+}\left(a^{2}-1\right)}{2 r}\right]}{\sqrt{1-\frac{r_{+}}{r}}},
$$

The meaning of this result is that an accelerating observer, who sits on the AS with $r$ distance far away from the center of the $\mathrm{CDBH}$ always detects thermal radiation with $T_{U}$. In other words, from the viewpoint of the accelerating observer, the space of the inertial observer in which it is filled with the quantized fields that make up a universe around CDBH looks like a state containing many particles in thermal equilibrium with $T_{U}$. It is noteworthy that the Unruh effect emerges from the local quantum fields [16]. In the Schwarzschild limit $\left(a^{2} \rightarrow \infty\right.$ with $\left.\gamma=1\right)$, the Unruh temperature $(22)$ reduces to

$$
T_{U} \rightarrow T_{U_{\text {Schw. }}}=\frac{M}{2 \pi r^{2}} \frac{1}{\sqrt{1-\frac{2 M}{r}}},
$$

which has the Newtonian limit at large distances $r \gg$

$$
\lim _{r \rightarrow \infty} T_{U_{\text {Schw. }}}=\frac{M}{2 \pi r^{2}}
$$


Since our aim is to attain the entropic force on the AS of the CDBH, the flux $\Phi_{A S}$ of the proper acceleration through the AS should be taken into account. To this end, we use the definition of $\Phi_{A S}$ given by [14]

$$
\Phi_{A S}=4 \pi \tilde{a} R^{2}
$$

from which it readily follows that

$$
\Phi_{A S}=\frac{4 \pi \gamma}{1+a^{2}} r^{-2 N} \frac{\left[1+\frac{r_{+}\left(a^{2}-1\right)}{2 r}\right]}{\sqrt{1-\frac{r_{+}}{r}}}
$$

Introducing the change of heat $\delta Q$ as

$$
\delta Q=\frac{1}{4 \pi} d \Phi_{A S}
$$

where $d \Phi_{A S}$ denotes the differential of the flux $\Phi_{A S}$ through the AS. Let us suppose that the proper acceleration $\tilde{a}$ is uniform on the AS when varying the flux $\Phi_{A S}$. This corresponds to $d \tilde{a}(r, M)=0$ such that the following relation automatically appears:

$$
d M=\frac{\left\{\left(a^{4}-1\right) M\left[\left(1+a^{2}\right)\left(3 a^{2}+1\right) M-2 a^{4} r\right]-a^{6} r^{2}\right\}}{r\left(1+a^{2}\right)^{2}\left[\left(a^{4}-1\right) M-a^{4} r\right]} d r
$$

Inserting this into Eq. (27), and making some algebra, we end up with the following equation

$$
\delta Q=\frac{2 \gamma N}{1+a^{2}} r^{-\frac{1}{1+a^{2}}} \frac{\left[1+\frac{r_{+}\left(a^{2}-1\right)}{2 r}\right]}{\sqrt{1-\frac{r_{+}}{r}}} d r
$$

We may define the entropy as

$$
S_{A S}=2 \pi \gamma^{2} r^{2 N}
$$

whence its differential can be calculated as

$$
d S_{A S}=4 \pi \gamma^{2} N r^{\frac{a^{2}-1}{a^{2}+1}} d r
$$

Plugging the above equation into Eq. (29),

$$
\begin{aligned}
\delta Q & =\frac{r^{-N}}{2 \pi \gamma\left(1+a^{2}\right)} \frac{\left[1+\frac{r_{+}\left(a^{2}-1\right)}{2 r}\right]}{\sqrt{1-\frac{r_{+}}{r}}} d S_{A S} \\
& =T_{U} d S_{A S}=T_{A S} d S_{A S},
\end{aligned}
$$

This result represents a fact that the change of heat is balanced by the change of the entropy when fixing the temperature on the AS.

Finally, the entropic force, from Eq.(3), is obtained as,

$$
F_{A S}=\frac{m}{\gamma\left(1+a^{2}\right)} r^{-N} \frac{\left[1+\frac{r_{+}\left(a^{2}-1\right)}{2 r}\right]}{\sqrt{1-\frac{r_{+}}{r}}}
$$


which is exerting on the object with mass $m$ near to the AS of the CDBH having mass $M$. This result shows us that any object possessing mass $m$ will be under the influence of infinitely tidal force while it is close to the horizon, $r \rightarrow r_{+}$. At large distance limit, $r>>r_{+}, F_{A S}$ takes the following form

$$
F_{A S} \rightarrow F_{M N}=\frac{m}{\gamma} r^{-N}\left(\frac{1}{1+a^{2}}+\frac{M}{r}\right)
$$

One can easily see that the above equation takes the Newtonian force law (8) if $a^{2} \rightarrow \infty$, and $\gamma=1$ is chosen. That is why, we prefer to symbolize the large distance limit of $F_{A S}$ as $F_{M N}$, and designate it as modified Newtonian force. As illustrated in Fig.1, the value of the dilaton parameter $a$ is effective on $F_{M N}$, which is exerted by the mass $M$ of the $\mathrm{CDBH}$ on the object possessing mass $m$. Here, we considered a small object compared to the mass of the BH. It is clear that when $a \rightarrow 0, F_{M N}$ increases. This intriguing result might also be used to prove the existence of the dilaton fields in the future. On the other hand, it is observed that when $a$ gets higher values than zero, up to infinity, $F_{M N}$ behaves like the conventional Newtonian force. In the caption of Fig. 1, the values of the physical parameters are depicted.

\section{STATIC HOLOGRAPHIC SCREEN OF CDBH AND THE ENTROPIC FORCE}

In this section, we briefly review how one derives the entropic force on the static HS, which belongs to the CDBH (10). For this purpose, we first consider the following definition for nonzero component of the proper acceleration vector [17]

$$
a^{\mu}=\frac{1}{f} \xi^{\alpha} \nabla_{\alpha} \xi^{\mu}
$$

where $\xi^{v}$ is a timelike Killing vector. In order to define the concept of energy in the stationary spacetime, one might use

$$
E(V)=\frac{1}{4 \pi} \oint a^{\mu} n_{\mu} d A
$$

where $V$ is the bulk volume enclosed by spacelike hypersurface $S^{2}$ i.e. $\partial V=S^{2}$. In general, the nonzero component of the Killing vector for the outer region of the $\mathrm{CDBH}\left(r>r_{+}\right)$is given by $\xi^{\alpha}=[1,0,0,0]$. By using these quantities, we can easily evaluate the integral (36) and obtain the energy, which is defined on the HS of the CDBH as follows

$$
E_{H S}=\frac{\gamma}{1+a^{2}} r^{2-N} \frac{\left[1+\frac{r_{+}\left(a^{2}-1\right)}{2 r}\right]}{\sqrt{1-\frac{r_{+}}{r}}},
$$

One can easily verify that while $a^{2} \rightarrow \infty$ with $\gamma=1$, it reduces to

$$
E_{H S} \rightarrow \frac{M}{\sqrt{1-\frac{r_{+}}{r}}},
$$

which is the energy of the HS for the Schwarzschild BH. Namely, our result (37) is consistent with the HS energy result reported in [10]. When an observer is at rest and located at coordinate $r$ with respect to CDBH spacetime, $E_{H S}$ corresponds to the energy of the gravitational field, which is measured by that observer. After imposing the local equipartition rule

$$
E_{H S}=2 S_{H S} T_{H S}
$$

where $S_{H S}$ indicates the entropy on the HS located at $r$, where is outside of the horizon. $S_{H S}$ is found by

$$
S_{H S}=\pi R^{2},
$$


Thus, by using of Eq. (39) one can obtain the temperature on the HS of CDBH as

$$
T_{H S}=\frac{1}{2 \pi \gamma\left(1+a^{2}\right)} r^{-N} \frac{\left[1+\frac{r_{+}\left(a^{2}-1\right)}{2 r}\right]}{\sqrt{1-\frac{r_{+}}{r}}},
$$

Considering Eq.(32), we see that $T_{H S}=T_{A S}$. Namely, the bulk temperature $T_{A S}$ is exactly equal to the HS temperature $T_{H S}$.

Finally, inserting Eq.(41) into Eq. (3), we attain the entropic force on the object $m$ near the HS as

$$
F_{H S}=2 \pi m T_{H S}=\frac{m}{\gamma\left(1+a^{2}\right)} r^{-N} \frac{\left[1+\frac{r_{+}\left(a^{2}-1\right)}{2 r}\right]}{\sqrt{1-\frac{r_{+}}{r}}},
$$

which means that the entropic force $(33)$ is recovered impeccably: $F_{H S}=F_{A S}$.

As a final remark for this section, we would like to highlight that temperature $T_{H S}$, which belongs to an spacetime (10) with unusual asymptotics is obtained by the using of the local equipartition rule. Thence, the entropic force (3) is read for those unusual asymptotical CDBHs. All deserved limits of that entropic force (42) can be easily verified. Consequently, the procedure followed up to obtain the entropic force shows us that it is not only applicable to AF BHs, but it could be used for NAF BHs as well.

\section{STRETCHED HORIZON OF CDBH AND THE ENTROPIC FORCE}

In this section, the SH is considered as a HS, and we explore the form of the entropic force on the SH. The concept of SH [18] arouses interest because of its location. Its location is very close to the event horizon (near horizon limit of the $\mathrm{CDBH}$ or the Rindler space [19] of the $\mathrm{CDBH}$ ), and all temperatures on the SH that are obtained by different approaches become equivalent to each other. Through the section, the radial distance of the SH from the center of the CDBH is considered as $r=r_{+}+1 / r_{+}$in which $r_{+} \gg 1$. Briefly, the SH is located just above the event horizon.

The AS and its equivalent HS temperatures, which are represented by Eqs. (22) and (41) take the following forms on the SH.

$$
\begin{aligned}
T_{A S}^{S H} & =T_{H S}^{S H}=\frac{1}{4 \pi \gamma}\left(r_{+}\right)^{\frac{1}{1+a^{2}}}\left(1+\frac{1}{r_{+}^{2}}\right)^{-\frac{3 a^{2}+1}{2\left(1+a^{2}\right)}}\left[1+\frac{2}{r_{+}^{2}\left(1+a^{2}\right)}\right] \\
& \simeq \frac{1}{4 \pi \gamma}\left(r_{+}\right)^{\frac{1}{1+a^{2}}}\left[1-\frac{3\left(a^{2}-1\right)}{2 r_{+}^{2}\left(1+a^{2}\right)}\right]
\end{aligned}
$$

Since the near horizon limit of a $\mathrm{BH}$ is also known as the Rindler space of the considered $\mathrm{BH}$, the geometry of a SH can be described by a Rindler spacetime. Besides, the local Rindler temperature [20] is given by

$$
T_{L R}^{S H}=\left.\frac{\kappa(r)}{2 \pi \sqrt{-g_{00}}}\right|_{r=r_{+}+1 / r_{+}}=\left.\frac{f^{\prime}}{4 \pi \sqrt{f}}\right|_{r=r_{+}+1 / r_{+}}
$$

which yields

$$
\begin{aligned}
T_{L R}^{S H} & =\frac{1}{2 \pi \gamma\left(1+a^{2}\right)}\left(r_{+}\right)^{\frac{1}{1+a^{2}}}\left[\frac{1}{2}\left(1+a^{2}\right)+\frac{1}{r_{+}^{2}}\right] \\
& \simeq \frac{1}{4 \pi \gamma}\left(r_{+}\right)^{\frac{1}{1+a^{2}}}
\end{aligned}
$$

As envisioned before, all temperatures on the $\mathrm{SH}$, which are obtained by using different approaches are equal to each other in the leading order: 


$$
T_{A S}^{S H}=T_{H S}^{S H}=T_{L R}^{S H}=\frac{1}{4 \pi \gamma}\left(r_{+}\right)^{\frac{1}{1+a^{2}}},
$$

As it can be seen from the above equation, unless $a \rightarrow \infty$, the SH temperature will depend on the quasilocal mass $M$.

On the other, the definition of the local energy is given by

$$
E_{L}=\frac{M}{\sqrt{-g_{00}}},
$$

In the Rindler space, the foregoing takes the following form

$$
\begin{aligned}
E_{L R}^{S H} & \simeq \gamma M\left(r_{+}\right)^{N} \\
& =\frac{N A_{E H}}{8 \pi \gamma}\left(r_{+}\right)^{\frac{1}{1+a^{2}}}
\end{aligned}
$$

It is possible to obtain the same result when Eq. (37) is used for in defining the local energy of the holographic screen on the stretched horizon $-E_{L R}^{S H}=E_{H S}^{S H}$. Significantly, one can easily verify that the equipartition of energy $E_{H S}^{S H}=\frac{1}{2} \tilde{N}^{S H} T_{H S}^{S H}$ is satisfied. However, the number of bits stored on the holographic screen is found as $\tilde{N}^{S H}=$ $N A_{E H}$. When comparing this result with Eq. (5), we see that the proportionality constant $c=N$, which changes between $0^{+}$and 1 i.e., $0^{+}<c<1$ depending on the value of the dilaton parameter $a$. In other words, the dilaton parameter $a$ scales the total information stored on the screen such that it reaches its maximum when $a \rightarrow \infty$, which corresponds to the vanishing of the dilaton field or to the AF spacetime. In terms of the Bekenstein-Hawking entropy

$$
S_{B H}=\frac{A_{E H}}{4},
$$

one can easily see that $\tilde{N}^{S H}=4 N S_{B H}$. After imposing the first law of thermodynamics

$$
d E_{H S}^{S H}=T_{H S}^{S H} d S^{S H}
$$

we see that the entropy on the SH should be

$$
S^{S H}=\frac{1+2 a^{2}}{1+a^{2}} S_{B H}
$$

Obviously, the factor $\frac{1+2 a^{2}}{1+a^{2}}$ always prevents the equality of both entropies. Nevertheless, in the case of ultrahigh dilaton field, corresponding to the limit of $a \rightarrow 0^{+}, S^{S H}$ and $S_{B H}$ become almost same. Conversely, while $a \rightarrow \infty$, $S^{S H}$ becomes double of $S_{B H}$, as can be seen in [10]. Hence, one can remark that depending on the dilaton parameter $a$, the rate $\frac{S^{S H}}{S_{B H}}$ changes in the manner that $1<\frac{S^{S H}}{S_{B H}}<2$.

Finally, according to our established entropic force derivation, it is now necessary to substitute Eq. (46) into Eq. (3). Thus, the entropic force on the SH yields

$$
\begin{aligned}
F_{S H} & =2 \pi m\left(T_{A S}^{S H}=T_{H S}^{S H}=T_{L R}^{S H}\right) \\
& =\frac{1}{2 \gamma} m\left(r_{+}\right)^{\frac{1}{1+a^{2}}} \\
& =m \tilde{a}_{S H}
\end{aligned}
$$

where $\tilde{a}_{S H}=\frac{1}{2 \gamma}\left(r_{+}\right)^{\frac{1}{1+a^{2}}}$ is the proper acceleration on the SH. Alternatively, $\tilde{a}_{S H}$ can alternatively be obtained by taking the near horizon limit $\left(r=r_{+}+1 / r_{+}\right)$of Eq. (21). 


\section{CONCLUSION}

After making the remarkable connections between gravity and thermodynamics, we showed that whenever the temperature on a holographic screen is read precisely, it is easy to calculate the entropic force by using $F=2 \pi m T$. In addition to this, it is also shown that Verlinde's entropic force formalism is applicable to the CDBHs, which are neither AF nor NAF spacetimes. As a result, we support the Verlinde's arguments, which imply that gravity is not a fundamental force but emergent.

In this paper, we introduced three possible distinct surfaces (or screens), which are separately being a candidate for the HS of a CDBH. Those are the AS, the static HS and the SH or the Rindler spacetime of the CDBH. Among them, the SH is the special one. Because, the SH is located at such a specific place that when all the other obtained temperatures are recalculated for the location of the SH, they all, and thus their corresponding entropic forces, become equivalent. On the other hand, the entropic forces obtained by using of the AS and of the HS reduce to the entropic force of the Schwarzschild BH when the dilaton field is terminated, and subsequently to the Newton's force law at large distances. Dependence of the entropic force of a small object (compared with the mass $M$ of the BH) having mass $m$ on the dilaton parameter at large distances is graphically illustrated. The graph shows us that a distant observer may feel the gravity of the CDBH higher than the Newton's force if the strength of the dilaton field increases. The latter result might provide a clue to experimentally test the concept of entropic force, or even to detect the dilaton fields.

Finally, we should say that the discussions on the Verlinde's arguments have been continuing without pausing, even day by day one can see many new studies, which are referring the Verlinde's work [2] pro and con (see for instance [21]). Although we sustain that our analysis is not adequate to fully understand the entropic force, here our ultimate aim is to make a contribution to the literature that the derivation of the entropic force according to the Verlinde's arguments is also possible in the spacetimes with unusual asymptotics. Moreover, we would like to keep the topic fresh. Because we believe that even the Verlinde's arguments turn out to be in the wrong, hashing the topic of the entropic force over with other researchers working in the same field will guide us for better understanding the origin of gravity.

\section{Acknowledgments}

I am grateful to Professor M. Halilsoy for fruitful discussions and suggestion of studying on the theory of emergent gravity.

[1] A. Einstein, Preuss. Akad. Wiss. Berlin, Sitzbr., 778-786 (1915); 844-847 (1915).

[2] E.P. Verlinde, arXiv: 1001.0785.

[3] J.D. Bekenstein, Phys. Rev. D 9, 3292 (1974); 23, 287 (1981).

[4] S. W. Hawking, Comun. Math. Phys. 43, 199 (1975); 46, 206 (E) (1976).

[5] G. t'Hooft, arXiv: 9310026.

[6] L. Susskind, J. Math. Phys. 36, 6377 (1995); E. Witten, arXiv: 0106109; T. Padmanabhan, arXiv: 0606061; L. Susskind and J. Lindesay, Black Holes, Information and the String Theory Revolution (World Scientific, Singapore, 2005).

[7] T. Padmanabhan, arXiv: 0912.3165; Class. Quantum Grav. 21, 4485 (2004).

[8] Y.-X. Liu, Y.-Q. Wang, and S.-W. Wei, Class. Quantum Grav. 27, 185002 (2010); H. Wei, Phys. Lett. B 692, 167 (2010); P. Nicolini, Phys. Rev. D 82, 044030 (2010).

[9] K.C.K. Chan, J.H. Horne and R.B. Mann, Nucl. Phys. B 447, 441 (1995).

[10] Y.S. Myung and Y.-W. Kim, Phys. Rev. D 81, 105012 (2010).

[11] D. Garfinkle, G.T. Horowitz, and A. Strominger, Phys. Rev. D 43, 3140 (1991); 45, 3888 (E) (1992).

[12] J.D. Brown and J.W. York, Phys. Rev. D 47 (1993) 1407; J.D. Brown, J. Creighton and R.B. Mann, Phys. Rev. D 50, 6394 (1994).

[13] G. Clément and C. Leygnac, Phys.Rev. D 70, 084018 (2004); S. H. Mazharimousavi, I. Sakalli, and M. Halilsoy, Phys. Lett. B 672, 177 (2009).

[14] J. Mäkelä, arXiv: 0805.3955.

[15] W. Unruh, Phys. Rev. D 14, 870 (1976); Ann. N.Y. Acad. Sci. 302, 186 (1977).

[16] A. Gill, D. Singleton, V. Akhmedova, and T. Pilling, Am. J. Phys. 78, 685 (2010).

[17] Y. Tian and X.-N. Wu, Phys. Rev. D 81, 104013 (2010).

[18] L. Susskind, L. Thorlacius, and J. Uglum, Phys. Rev. D 48, 3743 (1993).

[19] N. Kaloper, M. Kleban, A. Lawrence, S. Shenker, and L. Susskind, J. High Energy Phys. 11, 037 (2002).

[20] T. Padmanabhan, Class. Quantum Grav. 19, 5387 (2002). 
[21] C. Gao, Phys. Rev. D 81, 087306 (2010); A. Kobakhidze, Phys. Rev. D 83, 021502 (2011), arXiv:1009.5414 [hep-th].

Figure Caption:

Figure 1: Entropic Force $F_{M N}$ versus the dilaton parameter $a$ for the CDBHs. The plot is governed by Eq. (34). The physical parameters in Eq. (34) are chosen as follows: $\gamma=1, M=1$ and $m=1 \times 10^{-6}$. 
This figure "FIG1.jpg" is available in "jpg" format from: http://arxiv.org/ps/1103.1728v2 